\title{
Actualités en phytothérapie
}

\author{
P. Goetz \\ (C) Lavoisier SAS 2018
}

\section{La prêle : pas uniquement la silice}

Résumé : Lorsqu'on évoque la prêle, on se pose la question suivante : est-ce la silice (c'est la plante la plus riche en silice) qui rend l'Equisetum efficace dans des désordres articulaires ? Une équipe germano-franco-suisse [1] a tenté de répondre à la question en utilisant l'activité basée sur la cytométrie, son profil de fonctionnalité sur les lymphocytes humains primaires ainsi que le dosage de cytokines par une méthode Elisa. Les préparations d'Equisetum arvense suppriment l'activation et la prolifération lymphocytaire par un mécanisme passant par l'interleukine-IL2. L'effet augmente en fonction de la teneur en silice des décoctions. La polyfonctionnabilité des lymphocytes est aussi influencée, et ce, par une régulation à la baisse de l'IFN- $\gamma$. Grâce à l'HPLCUV-MS, on remarque que l'isoquercitrine contenue par la prêle a une action immunosuppressive. En conclusion, autant la silice que cet isoquercitrine sont des substances participant à l'activité thérapeutique de la prêle.

Commentaires : Nous savions déjà que la prêle inhibe la prolifération des cellules T sans induction d'apoptose ou de nécrose, par inhibition de l'activation des lymphocytes, en diminuant l'expression des récepteurs de surface CD69 et IL-2 et la production d'IL-2 intracellulaire [2]. La présente expérimentation in vitro voudrait que la silice agisse dans ce sens. Il est connu que la quercitrine intensifie avec un apport de protéine l'hypertrophie des fibres du muscle plantaire chez la souris [3] soumise au port d'un poids. La contracture musculaire est bénéfique pour les articulations : serait-ce un effet mécanique qu'induisent les deux substances ?

\section{Références}

1. Steinborn C, Potterat O, Meyer U, et al (2017) In Vitro Anti-inflammatory Effects of Equisetum arvense Are Not Solely Mediated by Silica. Planta Med 18:84

P. Goetz $(\bowtie)$

Dumenat de phytothérapie, Paris-XIII,

F-93017 Bobigny cedex, France

e-mail : paul.goetz@wanadoo.fr
2. Gründemann C, Lengen K, Sauer B, et al (2014) Equisetum arvense (common horsetail) modulates the function of inflammatory immunocompetent cells. BMC Complement Altern Med 14:283

3. Kohara A, Machida M, Setoguchi Y, et al (2017) Enzymatically modified isoquercitrin supplementation intensifies plantaris muscle fiber hypertrophy in functionally overloaded mice. J Int Soc Sports Nutr 14:32

\section{L'inflammation pulmonaire et le plantain}

Résumé : Le plantamajoside (Fig. 1) est un constituant majeur de Plantago asiatica L. Les auteurs chinois [1] ont montré que cette substance a un fort effet anti-inflammatoire et qu'il pouvait être utilisé dans des affections pulmonaires. Ils ont mis en lumière l'effet anti-inflammatoire de l'atteinte aiguë du tissu pulmonaire soumis au lipopolysaccharide (LPS) chez la souris et dans les cellules RAW264.7 (macrophage murin). Le plantamajoside améliore l'état d'une lésion pulmonaire par le LPS, son état histopathologique et l'activité de la myéloperoxydase. Les essais aux tests qPCR (réaction en chaîne par polymérase) et Elisa démontrent que le plantamajoside supprime la production des IL-1 $\beta$, IL-6 et TNF- $\alpha$ de manière dose-dépendante. Il inhibe aussi le Toll Like Receptor 4 (récepteur membranaire retrouvé sur les macrophages ainsi que sur certains adipocytes qui reconnaît des motifs moléculaires tels que les lipopolysaccharides bactériens ou endotoxine). Le plantamajoside inhibe la phosphorylation du I $\kappa \mathrm{B} \alpha$ (facteur nucléaire de l'amplificateur du gène du polypeptide léger (kappa) et les kinases p65, p38, JNK et ERK. Les auteurs estiment intéressant de poursuivre les études sur le plantamajoside dans l'inflammation pulmonaire souvent difficile à soigner.

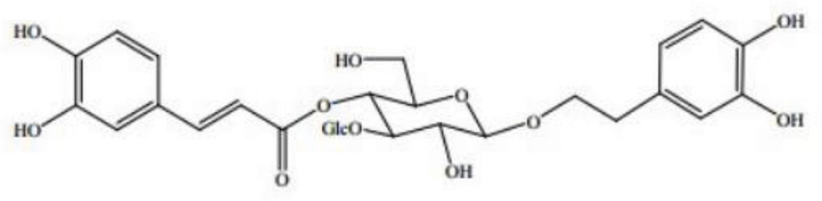

Fig. 1 Structure du plantamajoside 
Commentaire : Ce plantain asiatique qui pousse dans les zones montagneuses du Japon, de la Chine et de la Corée ressemble beaucoup au plantain européen. Il contient donc du plantamjoside qui par ailleurs a un effet antitumoral dans le cancer mammaire en inhibant l'acticité des métallopeptidases matricielles MMP9 et MMP2 (Pei). Le plantamajoside est un phényléthanoïde. Proche de l'actéoside ou du verbascoside, le plantamajoside n'a pas besoin d'être synthétisé ; une dose suffisante et efficace de l'extrait de Plantago asiatica peut être utile au traitement.

\section{Références}

1. Wu H, Zhao G, Jiang K (2016) Plantamajoside ameliorates lipopolysaccharide-induced acute lung injury via suppressing NF- $\mathrm{\kappa B}$ and MAPK activation. Int Immunopharmacol 35:315-22

2. Pei S, Yang X, Wang H (2015) Plantamajoside, a potential anti-tumor herbal medicine inhibits breast cancer growth and pulmonary metastasis by decreasing the activity of matrix metalloproteinase-9 and -2 BMC. Cancer 15:965

\section{Du souci pour la parodontite}

Résumé : La parodontite s'accompagne fréquemment d'une réduction de la capacité antioxydante des patients qui en sont atteints avec perte de masse osseuse. Une équipe brésilienne a étudié l'apport possible d'un extrait de souci (Calendula officinalis) sur cette pathologie. Les souris Wistar ont reçu une solution neutre, une solution salée ou l'extrait de souci. Les rats perçurent de la solution salée ou de l'extrait de souci à la dose de $(90 \mathrm{mg} / \mathrm{kg})$ trois minutes avant une ligature pendant 11 jours. L'examen immunohistochimique du périodonte à 11 jours de ligature montre qu'elle a entraîné une perte osseuse, un effondrement des fibres de collagène, une sensibilité immunologique de la protéine DKK-1 et de la glycoprotéine WNT 10B et de l'expression de la $\beta$-caténine (ayant un rôle dans l'adhésion cellulaire, la signalisation cellulaire et la transcription des protéines). Tous les résultats s'inversent à une dose de $90 \mathrm{mg} / \mathrm{kg}$. Le souci réduit donc le stress oxydatif avec perte osseuse et préserve les fibres de collagènes en passant par la voie du WNT10B, gène de l'ontogenèse et de processus de développement.

Commentaires : Le souci (Calendula officinalis L.) est depuis toujours un traitement des inflammations et allergies de la bouche. Il s'attaque aussi modérément aux microbes qui s'accumulent dans ces lésions du parodonte. Le travail ci-dessus permet cependant de voir que le souci a aussi d'autres pouvoirs passant par le système immunogénétique.

\section{Référence}

1. Lima MDR, Lopes AP, Martins C, et al (2017) The Effect of Calendula officinalis on Oxidative Stress and Bone Loss in Experimental Periodontitis. Front Physiol 8:440

\section{Un remède de médecine traditionnelle chinoise pour le psoriasis}

Résumé : Le Yin xie ling est une formule chinoise utilisée dans le traitement du psoriasis depuis plus de 20 ans. Le mélange PSORI-CM01 est une préparation selon une simplification de la formule originale. Le PSORI-CM01 se compose de sept plantes : Rhizome de curcuma (E Zhu), Radix Paeoniae Rubra (Chi Shao), Sarcandra glabra (Zhong-Jie Feng), Radix Arnebiae (Zi Cao), Rhizoma Smilacis Glabrae (Tu-Fu Ling), Fructus mume (Wu Mei), Radix glycyrrhizae (Gan Cao). Le Fructus mume (Wu Mei) est le fruit de la prune noire. L'unité médicale du Guangdong (Chine) a décidé de vérifier les effets avec une étude clinique randomisée sur 12 semaines. La prise de PSORI-CM01 entraîne une première manifestation qui est la réduction de l'aire de psoriasis et de l'index de sévérité. En second, on trouve une amélioration de l'échelle visuoanalogique et du questionnaire de l'impact sur la qualité de vie.

Commentaire : Le rhizome de Curcuma longa est connu comme antioxydant et anti-inflammatoire (de même que dans le domaine immunologique), la racine de pivoine rouge est antirhumatismale et agit sur le cerveau (striatum), Sarcandra glabra est antirhumatismal, Arnebia agit sur la chaleur dans le sang et en cas d'oreillon ainsi qu'en externe sur les plaies, le rhizome de Smilax glabra réchauffe le yang du Rein et réduit les gonflements, le fruit de la prune est astringente du poumon, stimule les fluides et améliore la colite due à l'ascaris, enfin la racine de réglisse est anti-inflammatoire. La préparation chinoise selon la pharmacologie occidentale renferme donc des substances qui peuvent agir sur une pathologie dermato-immunologique comme le psoriasis.

\section{Référence}

1. Deng J, Yao D, Lu C, et al (2017) Oral Chinese herbal medicine for psoriasis vulgaris: protocol for a randomised, double-blind, double-dummy, multicentre clinical trial. BMJ Open 7:e014475

\section{Toxicologie en phytothérapie}

\section{Interaction entre racine de céleri et venlafaxine}

Résumé : La racine de céleri appartient à un groupe de plantes classées dans la famille des Ombellifères, qui contient 
des phytoestrogènes. Une femme de 52 ans a développé un syndrome de désordre bipolaire par l'interaction entre la venlafaxine et le céleri. Des extraits de céleri pris par la patiente pour des problèmes de ménopause l'ont mise dans un état de manie bipolaire avec une concentration plasmatique de venlafaxine importante. Le fait de retirer le céleri de son traitement a diminué la symptomatologie. Les auteurs estiment que c'est la prise de céleri et non l'association de venlafaxine avec du millepertuis qui a induit cet effet secondaire, et ce double traitement est maintenu.

\section{Référence}

1. Khalid Z, Osuagwu FC, Shah B, et al (2016) Celery root extract as an inducer of mania induction in a patient on venlafaxine and St John's Wort. Postgrad Med 128:682-3

\section{Effets psychotiques du fruit de Garcinia cambogia}

Résumé : Le fruit de Garcinia cambogia de l'Asie du SudEst est de plus en plus populaire en tant que médication de la gestion du poids. L'acide hydroxycitrique est le principal ingrédient actif qui montre des propriétés sérotoninergiques et muscariniques par inhibition de la recapture sélective de la sérotonine et de l'acétylcholinestérase. Il faut signaler une jeune femme adulte sans antécédents de trouble bipolaire qui a développé manie et psychose environ une semaine après l'initiation d'un traitement par Garcinia cambogia associé au complément alimentaire Cleanse and Detox ${ }^{\mathrm{TM}}$ fabriqué par Apex Vitality Health qui réduit l'appétit et accélère le métabolisme. Les symptômes indésirables ont été : humeur expansive, agitation psychomotrice, discours désorganisé, vol d'idées, grandiosité, délires et hallucinations auditives. Sous lithium et quétiapine, la patiente a été stabilisée avec une humeur normalisée au bout de huit jours. Sept rapports antérieurs associant (hypo-) manie et/ou psychose à la consommation de Garcinia cambogia ont été publiés. Le symptôme psychotique apparaît entre une et huit semaines. Il est important que les urgences médicale et psychiatrique soient au courant de ce type d'effet secondaire.

\section{Référence}

1. Nguyen DC, Timmer TK, Davison BC, et al (2017) Possible Garcinia cambogia-induced mania with psychosis: a case report. J Pharm Pract 897190017734728

\section{Un cas de vasoconstriction cérébrale par l'eucalyptus}

Résumé : A El Paso (TX, États-Unis) une femme de 42 ans s'est présentée aux urgences avec depuis deux semaines une sensation de picotement sur le côté droit de son corps, des céphalées holocrâniennes sévères palpitantes progressives irradiant dans le cou, avec nausées associées, photophobie et phonophobie. Pas de notion de traumatisme cérébral ni de prise de médicaments. La patiente se traite par contre pour une sinusite congestive avec des vapeurs de feuilles d'eucalyptus. L'imagerie cérébrale pratiquée aux urgences indique un syndrome de vasoconstriction cérébrale réversible (ou syndrome de Call-Fleming) avec hémorragie sousarachnoïdienne. L'utilisation de « simples » vapeurs d'eucalyptus peut donc induire un syndrome sérotoninergique avec vertiges, diarrhée et vasoconstriction cérébrale.

\section{Référence}

1. Lapid D, Qureshi MA, Quresh IA, et al (2017) Possible Reversible Cerebral Vasoconstriction Syndrome Associated with Eucalyptus: Case Report. J Vasc Interv Neurol 9:17-20 